\title{
EXACT SOLUTION OF SPAN-WISE FLUCTUATING MHD CONVECTIVE FLOW OF SECOND GRADE FLUID THROUGH POROUS MEDIUM IN A VERTICAL CHANNEL WITH HEAT RADIATION AND SLIP CONDITION
}

\author{
Krishan Dev Singh \\ Wexlow, Lower Kaithu, Shimla 171003, India. \\ E-mail: kdsinghshimla@gmail.com
}

(Received November 14, 2014)

\begin{abstract}
The magnetohydrodynamic (MHD) convective flow of a viscoelastic, incompressible and electrically conducting fluid through a porous medium filled in a vertical channel is analyzed. The channel plate at $y^{*}=-\frac{d}{2}$ is subjected to a slip-flow condition and the other at $y^{*}=+\frac{d}{2}$ to a no-slip condition. The temperature of the plate at $y^{*}=+\frac{d}{2}$ with no-slip condition is assumed to be varying both in space and time. The temperature difference of the walls of the channel is assumed high enough to induce heat transfer due to radiation. A magnetic field of uniform strength is applied perpendicular to the planes of the channel walls. The magnetic Reynolds number is assumed very small so that the induced magnetic field is neglected. It is also assumed that the conducting fluid is optically-thin gray gas, absorbing/ emitting radiation and non-scattering. Exact analytical solutions of the non-linear partial differential equations governing the flow problem are obtained. The velocity field, the temperature field, the amplitude and the phase angle of the skin friction and the heat transfer coefficient are shown graphically and their dependence on the various flow parameters is discussed in detail.
\end{abstract}

Keywords: Slip-condition, second grade, MHD, span-wise sinusoidal temperature, convective flow, porous medium, heat radiation.

\section{INTRODUCTION}

In recent years, the interest in the study of flows of non-Newtonian fluids through porous medium has grown considerably because of their applications in engineering. This is mainly due to their several applications in petroleum industry, manufacturing and processing of foods, paper industry and many other industrial applications, for example filtration processes, biomechanics, packed bed reactors, insulation system, ceramic processing, enhanced oil recovery, chromatography and many others. SINGH and SINGH [1] studied MHD flow of a dusty viscoelastic liquid through a porous medium between two inclined parallel plates. HAYAT et al. [2] discussed analytical solution for MHD transient rotating flow of a second grade fluid in a porous space. TIWARI and RAVI [3] studied analytically the transient rotating flow of a second grade fluid in a porous medium. Heat transfer aspect to MHD oscillatory viscoelastic flow in a channel filled with porous medium is presented by Choudhary and Das [4]. GHOSH and SHIT [5] analyzed mixed convection MHD flow of 
viscoelastic fluid in a porous medium past a hot vertical plate. CHOUDHURY et al. [6] investigated visco-elastic free convective flow past a vertical porous plate through a porous medium with suction and heat source.

The problems of flow of non-Newtonian fluids offer varied challenges to applied mathematicians, numerical analysts and modelers in developing suitable algorithms for computing the flows. From literatures, the non- Newtonian fluids are principally classified on the basis of their behavior in shear. A fluid with a linear relationship between the shear stress and the shear rate, giving rise to a constant viscosity, is always characterized to be a Newtonian fluid. The equations that describe flows of Newtonian fluid are the Navier-Stokes equations. The exact solutions for Navier- Stokes equation are rare. Based on the knowledge of solutions to Newtonian fluid, the different fluids can be extended, such as Maxwell fluid, Voigt fluid, Oldroyd-B fluid, Rivlin-Ericksen fluid or power-law fluid. RAPTIS and TAKHAR [7] studied heat transfer from flow of an elastico-viscous fluid. HAYAT et al. [8] obtained solution of MHD flows of an Oldroyd-B fluid. MEHTA and RAO [9, 10] discussed buoyancy induced flow of non-Newtonian fluids over a non-isothermal horizontal plate embedded in a porous medium and with non-uniform surface heat flux. Due to the complexity of fluids, several constitutive equations of non-Newtonian fluids have been proposed in the literature. Amongst these there is a subclass of non-Newtonian fluids namely the second grade fluids for which one can reasonably hope to obtain analytical solution. In the case of differential type fluids, the equations of motion are one order higher than the Navier-Stokes equations and, thus, the adherence boundary condition is insufficient to determine the solution completely (see refs. HAYAT et al. [11, 12] for a detailed discussion of the relevant issues). Because of this fact equations governing flow of non-Newtonian fluids are much more complicated. Therefore, the class of exact solutions further narrowed down for non-Newtonian fluids. RAJGOPAL and GUPTA [13] obtained an exact solution for the flow of a non-Newtonian fluid past an infinite porous plate. Another exact solution of non-Newtonian fluid flows with prescribed vorticity is obtained by LABROPULU [14]. FETECAU and ZIEREP [15] presented a study on a class of exact solutions of the equations of motion of a second grade fluid. An exact solution of flow problem of a second grade fluid through two porous walls is arrived at by ARIEL [16]. KHAN et al. [17] obtained new exact solutions for an Oldroyd-B fluid in a porous medium. SINGH [18] analyzed another exact solution of viscoelastic mixed convection MHD oscillatory flow through a porous medium filled in a vertical channel.

The wall slip flow is another very important phenomenon that is widely encountered in this era of industrialization. It has numerous applications, for example in lubrication of mechanical devices where a thin film of lubricant is attached to the surface slipping over one another or when the surfaces are coated with special coatings to minimize the friction between them. By lubricating or coating the solid surface the fluid particles adjacent to it no longer move with the velocity of the surface but has a finite tangential velocity and, hence slips along the surface. TICHY [19] analyzed non-Newtonian lubrication with the convected Maxwell model. A number of scholars have shown their interest in the phenomenon of slip-flow regime due to its wide ranging applications. MARQUES et al. [20] have considered the effect of the fluid slippage at the plate for Couette flow. RHODES and RoulEAU [21] studied the hydrodynamic lubrication of partial porous metal bearings. The problem of the slip-flow regime plays a very important role in modern science, technology and vast ranging industrialization. In view of the practical applications of the slip-flow regime it remained of paramount interest for several scholars e.g. Sharma and Chaudhary [22]; ShARma [23]; JAIN and GUPTA [24]. KHALED and VAFAI [25] obtained exact solutions of oscillatory Stokes 
and Couette flows of Newtonian fluids under slip flow condition. MEHMOOD and ALI [26] also obtained an exact solution for the unsteady MHD oscillatory flow of a viscous fluid in a planer channel to study the effect of slip condition. Recently, SINGH [27] studied an oscillatory MHD forced convection flow of an electrically conducting, viscous incompressible fluid through a porous medium in a vertical channel under slip condition. He [28] further obtained an exact solution of an oscillatory fully developed MHD convection flow through a porous medium in a vertical porous channel in slip flow regime.

A number of studies have also appeared in the literature for the flows of non-Newtonian fluids in slip-flow regime. HAYAT et al. [29] studied slip flow and heat transfer of a second grade fluid past a stretching sheet through a porous space. SIDDIQUI et al. [30] analyzed effect of slip condition on unsteady flows of an Oldroyd-B fluid between parallel plates. AHMED and TALUKDAR [31] studied transient magnetohydrodynamic (MHD) flow of a visco-elastic fluid past an infinite vertical porous plate embedded in a porous medium with Hall current and slip condition in a rotating system. OJHA and PANDA [32] investigated the MHD flow of an elastico-viscous fluid in porous medium in a slip flow regime. SINGH [33] obtained an exact solution of an oscillatory MHD convective flow of a viscoelastic fluid through a porous medium in a rotating vertical channel in slip-flow regime with thermal radiation and Hall current. Recently, SINGH [34] MHD mixed convection visco-elastic slip flow through a porous medium in a vertical porous channel with thermal radiation.

The aim of the present study is to formulate and analyze the flow problem of viscoelastic (second grade), incompressible and finitely electrically conducting fluid through a porous medium bounded by two infinite vertical plates in the presence of heat radiation. The temperatures of channel plates with no-slip condition and with slip-condition respectively remain span-wise cosinusoidal and constant as shown in figure 1a,b. A magnetic field of uniform strength is applied transverse to the flow and the magnetic Reynolds number is assumed very small so that the induced magnetic field is neglected. It is also assumed that the conducting fluid is optically-thin gray gas, absorbing/ emitting radiation and non-scattering. An exact solution of the mathematical problem so formed is obtained and the final results for the velocity, temperature, shear stress and heat transfer coefficient in terms of their amplitudes and phase angles are discussed in the last section of the paper.

\section{BASIC EQUATIONS}

In order to derive basic equations for the problem under consideration following assumptions are made:

(i) The flow considered is unsteady and laminar between two infinite electrically nonconducting vertical plates.

(ii) The fluid is second order viscoelastic finitely conducting and with constant physical properties.

(iii)A magnetic field of uniform strength is applied normal to the flow.

(iv)The magnetic Reynolds number is taken small enough so that the induced magnetic field is neglected.

(v) Hall effect, electrical and polarization effects are neglected.

(vi)It is assumed that the fluid is optically-thin gray gas, absorbing/ emitting radiation and non-scattering.

Under these assumptions, we write hydromagnetic equations of continuity, motion and energy as:

$$
\nabla \cdot \boldsymbol{V}=0 .
$$




$$
\begin{gathered}
\rho\left[\frac{\partial \boldsymbol{V}}{\partial t}+(\boldsymbol{V} . \nabla \boldsymbol{V})\right]=\nabla \cdot \boldsymbol{T}+(\boldsymbol{J} \times \boldsymbol{B})+\boldsymbol{F}, \\
\rho c_{p} \frac{\partial T^{*}}{\partial t^{*}}=k \nabla^{2} T^{*}-\frac{\partial q^{*}}{\partial y^{*}},
\end{gathered}
$$

where in equation (2) $\mathbf{T}$ is Cauchy stress tensor and the constitutive equation derived by COLEMAN and NOLL [35] for an incompressible homogeneous fluid of second order is

$$
\boldsymbol{T}=-p_{1} I+\mu_{1} A_{1}+\mu_{2} A_{2}+\mu_{3} A_{1}^{2} .
$$

Here $-p_{1} I$ is the interdeterminate part of the stress due to constraint of incompressibility, $\mu_{1}$, $\mu_{2}$ and $\mu_{3}$ are the material constants describing viscosity, elasticity and cross-viscosity respectively. The kinematic $A_{1}$ and $A_{2}$ are the Rivelen Ericson constants defined as

$$
A_{1}=(\nabla \vec{V})+(\nabla \vec{V})^{T}, \quad A_{2}=\frac{d A_{1}}{d t}+(\nabla \vec{V})^{T} A_{1}+A_{1}(\nabla \vec{V})
$$

where $\nabla$ denotes the gradient operator and $\mathrm{d} / \mathrm{dt}$ the material time derivative. According to MARKOVITZ and COLEMAN [36] the material constants $\mu_{1}, \mu_{3}$ are taken as positive and $\mu_{2}$ as negative.

On the right hand side of equation (2) the last term $\boldsymbol{F}\left(=\rho g \beta T^{*}\right)$ accounts for the force due to buoyancy and the second last term is the Lorentz force due to magnetic field $\mathbf{B}$ given by

$$
\boldsymbol{J} \times \boldsymbol{B}=\sigma(\boldsymbol{V} \times \boldsymbol{B}) \times \boldsymbol{B} .
$$

Here $\mathbf{V}$ is the velocity vector, $\mathbf{B}$ is the magnetic field, $\mathbf{J}$ is the current density, $\rho$ is the density, $c_{p}$ is the specific heat at constant temperature, $\mathrm{k}$ is the thermal conductivity, $\sigma$ is electric conductivity and $\mathrm{q}^{*}$ is the heat radiation.

\section{FORMULATION OF THE PROBLEM}

We consider an unsteady flow of a viscoelastic, incompressible and electrically conducting fluid in a hot vertical channel filled with porous medium. A schematic diagram of the physical problem with span-wise cosinusoidal variation of plate temperature is shown in Figures 1a \&1b.

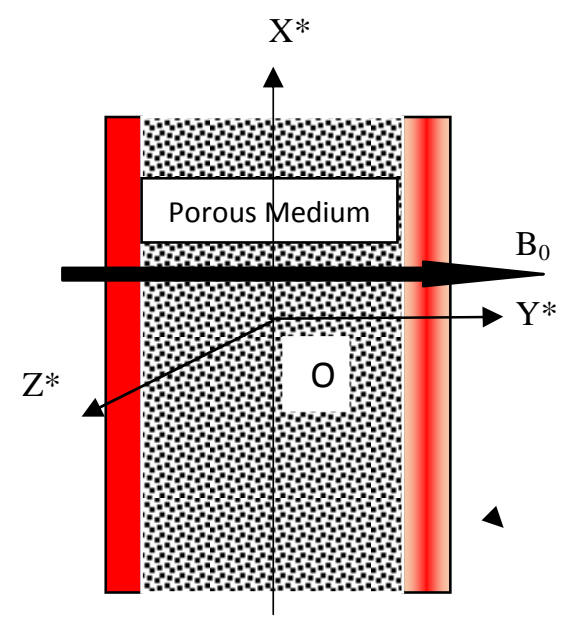

Fig. 1a. Hot vertical channel.

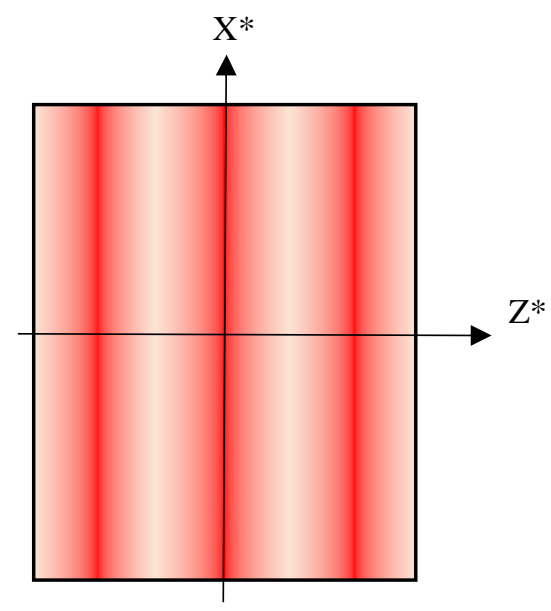

Fig. 1b. Spanwisecosinusoidal plate temperature. 
The two parallel stationary walls of the channel are distance ' $d$ ' apart. A Cartesian coordinate system $\left(\mathrm{X}^{*}, \mathrm{Y}^{*}\right)$ is chosen such that $\mathrm{X}^{*}$-axis directed upwards lies along the centerline of the channel and $\mathrm{Y}^{*}$-axis is perpendicular to the planes of parallel plates. A magnetic field $\mathrm{B}_{0}$ of uniform strength is applied transversely along $\mathrm{Y}^{*}$-axis. Since the walls of the channel are considered non-porous, thus, the integration of the continuity equation (1) implies that $\mathrm{v}^{*}=0$. All the physical quantities except pressure are independent of $x^{*}$ for this fully developed laminar flow in the infinite vertical channel. The temperature of the plate at $y^{*}=\frac{d}{2}$ varies span-wise cosinusoidally as

$$
T^{*}=T_{1}+\left(T_{2}-T_{1}\right) \cos \left(\frac{\pi z^{*}}{d}-\omega^{*} t^{*}\right) .
$$

Then taking into account the usual Boussinsq's approximation, the forced and free convection flow is governed by the following differential equations:

\section{Momentum equation;}

$$
\frac{\partial u^{*}}{\partial t^{*}}=-\frac{1}{\rho} \frac{\partial p^{*}}{\partial x^{*}}+\vartheta_{1} \nabla^{2} u^{*}+\vartheta_{2} \nabla^{2}\left(\frac{\partial u^{*}}{\partial t^{*}}\right)-\frac{\vartheta_{1}}{K^{*}} u^{*}-\frac{\sigma B_{0}^{2}}{\rho} u^{*}+g \beta\left(T^{*}-T_{1}\right),
$$

Energy equation;

$$
\frac{\partial T^{*}}{\partial t^{*}}=\frac{k}{\rho c_{p}} \nabla^{2} T^{*}-\frac{1}{\rho c_{p}} \frac{\partial q^{*}}{\partial y^{*}}
$$

where in momentum equation (8) the term on the L. H. S. is the inertial force and on the R. H. $\mathrm{S}$. the terms respectively represent imposed pressure gradient, viscous force, viscoelastic force, pressure drop across the porous matrix, Lorentz force due to magnetic field $\mathrm{B}_{0}$ and the buoyancy force due to temperature difference. In energy equation (9) the term on the L. H. S. is the heat due to convection and on the R. H. S. the terms respectively represent conduction heat and radiation heat.

The boundary conditions for this problem in slip flow regime are

$$
\begin{aligned}
& u^{*}=L \frac{\partial u^{*}}{\partial y^{*}}, \quad T^{*}=T_{1} \quad \text { at } y^{*}=-\frac{d}{2}, \\
& u^{*}=0, \quad T^{*}=T_{1}+\left(T_{2}-T_{1}\right) \cos \left(\frac{\pi z^{*}}{d}-\omega^{*} t^{*}\right) \quad \text { at } y^{*}=\frac{d}{2},
\end{aligned}
$$

where $=\left(\frac{2-r_{1}}{r_{1}}\right) L_{1}, \mathrm{~L}$ being the mean free path of the particle and $\mathrm{r}_{1}$ the Maxwell's reflection coefficient.

For the case of an optically thin gray gas the local radian $t$ is expressed by

$$
\frac{\partial q^{*}}{\partial y^{*}}=4 a^{*} \sigma^{*}\left(T^{* 4}-T_{1}^{4}\right)
$$

where $a^{*}$ is the mean absorption coefficient and $\sigma^{*}$ is the Stefan- Boltzmann constant.

We assume that the temperature differences within the flow are sufficiently small such that $\mathrm{T}^{* 4}$ may be expressed as a linear function of the temperature. This is accomplished by expanding $\mathrm{T}^{* 4}$ in a Taylor series about $\mathrm{T}_{1}$ and neglecting higher order terms, thus

$$
T^{* 4} \cong 4 T_{1}^{* 3} T^{*}-3 T_{1}^{4} \text {. }
$$

Substituting (13) into (12) and simplifying, we obtain 


$$
\frac{\partial q^{*}}{\partial y^{*}}=16 a^{*} \sigma^{*} T_{1}^{3}\left(T^{*}-T_{1}\right)
$$

Further, substitution of (14) into the energy equation (9) gives

$$
\frac{\partial T^{*}}{\partial t^{*}}=\frac{k}{\rho c_{p}} \nabla^{2} T^{*}-\frac{16 a^{*} \sigma^{*} T_{1}^{3}\left(T^{*}-T_{1}\right)}{\rho c_{p}} .
$$

Now introducing the following non-dimensional quantities

$$
x=\frac{x^{*}}{d}, y=\frac{y^{*}}{d}, z=\frac{Z^{*}}{d}, t=\omega^{*} t^{*}, \omega=\frac{\omega^{*} d^{2}}{\vartheta_{1}}, u=\frac{u^{*}}{U}, \theta=\frac{T^{*}-T_{1}}{T_{2}-T_{1}}, p=\frac{d p^{*}}{\rho \vartheta_{1} U},
$$

in equations (8), (15), (10) and (11) we obtain governing equations and the boundary conditions in dimensionless form as

$$
\begin{aligned}
& \omega \frac{\partial u}{\partial t}=-\frac{\partial p}{\partial x}+\nabla^{2} u+\gamma \omega \nabla^{2}\left(\frac{\partial u}{\partial t}\right)-\left(M^{2}+K^{-1}\right) u+G r \theta \\
& \omega \operatorname{Pr} \frac{\partial \theta}{\partial t}=\nabla^{2} \theta-N^{2} \theta
\end{aligned}
$$

with boundary conditions

$$
\begin{aligned}
& u=h \frac{d u}{d y}, \quad \theta=0, \quad \text { at } y=-\frac{1}{2}, \\
& u=0, \quad \theta=\cos (\pi z-t), \quad \text { at } \quad y=\frac{1}{2},
\end{aligned}
$$

where $\mathrm{U}$ mean axial velocity,

$\gamma=\frac{\vartheta_{2}}{d^{2}}$ is the viscoelastic parameter,

$G r=\frac{g \beta T_{0} d^{2}}{\vartheta_{1} U}$ is the Grashof number,

$\operatorname{Pr}=\frac{\vartheta_{1} \rho c_{p}}{k}$, is the Prandtl number,

$M=B_{0} d \sqrt{\frac{\sigma}{\rho \vartheta_{1}}}$, is the Hartmann number,

$K=\frac{K^{*}}{d^{2}}$ is the permeability of the porous medium,

$N=4 d \sqrt{\frac{a^{*} \sigma^{*} T_{1}^{3}}{k}}$, is the radiation parameter,

$h=\frac{L}{d}$, is the slip-flow parameter.

\section{SOLUTION OF THE PROBLEM}

In order to obtain the solution of this unsteady problem it is convenient to adopt complex variable notations for velocity, temperature and pressure. The real part of the solution will have physical significance. Thus, we write velocity, temperature and pressure as

$$
u(y, z, t)=u_{0}(y) e^{i(\pi z-t)}, \theta(y, z, t)=\theta_{0}(y) e^{i(\pi z-t)},-\frac{\partial p}{\partial x}=A e^{i(\pi z-t)},
$$

where $\mathrm{A}$ is a constant.

The boundary conditions in equations (19) and (20) can also be written in complex notations as

$$
u=h \frac{d u}{d y}, \quad \theta=0, \quad \text { at } \quad y=-\frac{1}{2},
$$




$$
u=0, \quad \theta=e^{i(\pi z-t)}, \quad \text { at } \quad y=\frac{1}{2} .
$$

Substituting expressions (21) into equations (17) and (18), we get

$$
\begin{aligned}
& l^{2} \frac{d^{2} u_{0}}{d y^{2}}-m^{2} u_{0}=-A-\operatorname{Gr} \theta_{0} \\
& \frac{d^{2} \theta_{0}}{d y^{2}}-n^{2} \theta_{0}=0
\end{aligned}
$$

where $=\sqrt{1-i \omega \gamma}, m=\sqrt{\pi^{2}+M^{2}+K^{-1}-i \omega\left(1+\gamma \pi^{2}\right)}$ and $n=\sqrt{\left(\pi^{2}+N^{2}-i \omega P r\right)}$ with transformed boundary conditions

$$
\begin{aligned}
& u_{0}=h \frac{d u_{0}}{d y}, \quad \theta_{0}=0 \quad \text { at } y=-\frac{1}{2}, \\
& \mathrm{u}_{0}=0, \quad \theta_{0}=1 \text { at } y=\frac{1}{2} .
\end{aligned}
$$

The ordinary differential equations (24) and (25) are solved under boundary conditions (26) and (27) and the solutions for the velocity and the temperature fields are obtained, respectively, as

$$
\begin{gathered}
u(y, z, t)=\left[\begin{array}{c}
\frac{A}{m^{2}}\left\{1-\frac{2 \sinh \left(\frac{m}{2 l}\right) \cosh \left(\frac{m}{l} y\right)+\frac{m}{l} h \cosh \left[\frac{m}{l}\left(y+\frac{1}{2}\right)\right]}{\left(\sinh \left(\frac{m}{l}\right)+\frac{m}{l} h \cosh \left(\frac{m}{l}\right)\right)}\right\}+\frac{G r}{\left(l^{2} n^{2}-m^{2}\right) \sinh (n)} \\
\left\{\begin{array}{c}
\frac{\sinh (n)\left[\sinh \left[\frac{m}{l}\left(y+\frac{1}{2}\right)\right]+\frac{m}{l} h \cosh \left[\frac{m}{l}\left(y+\frac{1}{2}\right)\right]\right]+n h \sinh \left[\frac{m}{l}\left(y-\frac{1}{2}\right)\right]}{\left(\sinh \left(\frac{m}{l}\right)+\frac{m}{l} h \cosh \left(\frac{m}{l}\right)\right)} \\
-\sinh \left[n\left(y+\frac{1}{2}\right)\right]
\end{array}\right\}
\end{array}\right] e^{i(\pi z-t),} \\
\theta(y, z, t)=\left\{\frac{\sinh \left[n\left(y+\frac{1}{2}\right)\right]}{\sinh (n)}\right\} e^{i(\pi z-t)} .
\end{gathered}
$$

From the velocity field in equation (28) we can obtain the skin-friction at the left wall, $\tau_{L}$, in terms of its amplitude $|F|$ and the phase angle $\varphi$ as

$$
\tau_{L}=\left(\frac{\partial u}{\partial y}\right)_{y=-\frac{1}{2}}=|F| \cos (\pi z-t+\varphi)
$$

where

$$
F_{r}+i F_{i}=\frac{A}{l m}\left\{\frac{\cosh \left(\frac{m}{l}\right)-1}{\sinh \left(\frac{m}{l}\right)+\frac{m}{l} h \cosh \left(\frac{m}{l}\right)}\right\}+\frac{G r}{\left(l^{2} n^{2}-m^{2}\right) \sinh (n)}\left\{\frac{m}{l}\left(\frac{\sinh (n)+n h \cosh \left(\frac{m}{l}\right)}{\sinh \left(\frac{m}{l}\right)+\frac{m}{l} h \cosh \left(\frac{m}{l}\right)}\right)-n\right\} .
$$

The amplitude and the phase angle of the skin-friction $\tau_{L}$ are respectively given by

$$
|F|=\sqrt{F_{r}^{2}+F_{i}^{2}}, \text { and } \varphi=\tan ^{-1}\left(\frac{F_{i}}{F_{r}}\right) .
$$

From the temperature field given in equation (29) the heat transfer coefficient $\mathrm{Nu}$ (Nusselt number) in terms of its amplitude $|H|$ and the phase angle $\psi$ can be obtained as

$$
N u=\left(\frac{\partial \theta}{\partial y}\right)_{y=-\frac{1}{2}}=|H| \cos (\pi z-t+\psi),
$$

where $H_{r}+i H_{i}=\frac{n}{\sinh n}$.

The amplitude $|H|$ and the phase angle $\psi$ of the heat transfer coefficient $\mathrm{Nu}$ (Nusselt number) are given by 


$$
|H|=\sqrt{H_{r}^{2}+H_{i}^{2}} \text { and } \psi=\tan ^{-1}\left(\frac{H_{i}}{H_{r}}\right) \text { respectively. }
$$

\section{RESULTS AND DISCUSSION}

An unsteady MHD convective flow of a viscoelastic fluid through a porous medium in a vertical channel under slip flow condition is analyzed. The closed form solutions for the velocity and temperature fields are obtained analytically and then evaluated numerically for different values of parameters appeared in the equations. To have better insight of the physical problem the variations of the velocity, temperature, skin-friction rate of heat transfer in terms of their amplitudes and phase angles with the parameters like viscoelastic parameter $\gamma$, Grashof number Gr, Hartmann number M, permeability of the porous medium K, Prandtl number Pr, radiation parameter $\mathrm{N}$, pressure gradient A and the frequency of oscillations $\omega$ are then shown graphically to assess the effect of each parameter.

The velocity variations with these parameters over the width of the channel are presented in Figure 2. The curve I (blue) corresponds the case of no-slip conditions at both the plates of the channel i.e. when the slip-flow parameter $h=0$. Curve II (green) represents the case of Newtonian fluid i.e. when viscoelastic parameter $\gamma=0$. Rests of all the curves are compared with curve III (red). Comparison of curves IV, VI, VIII and XI with red curve III clearly shows that the velocity increases with the increase of slip flow parameter $\gamma$, Grashof number Gr, permeability of the porous medium $\mathrm{K}$ and the favorable pressure gradient $\mathrm{A}$. The increasing slip-flow parameter clearly means that the increasing tangential velocity at the wall give rise to the velocity in the channel. The increase of velocity with increasing Grashof number, physically, means the increase of buoyancy force because of which velocity increases. The maximum of the velocity profiles for increased Grashof number shifts toward right half of the channel due to the greater buoyancy force in this part of the channel because of the presence of hotter plate otherwise the velocity remains parabolic with its maxima almost at the center of the channel with the increase of all other parameters. The increase of permeability of the porous medium $\mathrm{K}$ implies that the resistance posed by the porous matrix reduces and, thus, the velocity increases. It is also very natural that the flow will be faster for increased favorable pressure gradient. Similarly the comparison of rest of the curves namely V, VII, IX, X and XII with red curve III reveals that the velocity decreases with increasing viscoelastic parameter $\gamma$, Hartmann number M, Prandtl number Pr, radiation parameter $\mathrm{N}$ and the frequency of oscillations $\omega$. The velocity decreases with the increasing Hartmann number means that the flow retards with the increasing Lorentz force due to increasing magnetic field strength. Since the Prandtl number gives the relative importance of viscous dissipation to the thermal dissipation, therefore, for larger Prandtl number viscous dissipation is predominant and due to this velocity decreases. Thus, the velocity in the case of water $(\operatorname{Pr}=7)$ is less than that in the case of air $(\operatorname{Pr}=0.7)$.

The amplitude $|F|$ of the skin-friction against the frequency of oscillations is presented in Figure 3 for different sets of parameter values. In this figure comparison of curves IV, VI and IX with the dashed curve I (---) reveals that the amplitude increases with the increase of Grashof number Gr, permeability of the porous medium $\mathrm{K}$ and the pressure gradient parameter A. However, the comparison of curves II, III, V, VII and VIII shows that $|F|$ decreases with the increase of slip-flow parameter $h$, viscoelastic parameter $\gamma$, Hartmann number M, Prandtl number $\operatorname{Pr}$ and the radiation parameter N. The amplitude goes on decreasing with increasing frequency of oscillations $\omega$. The phase angle $\varphi$ of the skin-friction 
is presented in Figure 4 against the frequency $\omega$ of oscillations. The comparison of curves III, IV and VI with the dashed curve I (---) in this figure exhibits that the phase angle of the skinfriction increases with increase of viscoelastic parameter $\gamma$, Grashof number Gr, and permeability $\mathrm{K}$ of the porous medium while the phase angle decreases with the increase of slip-flow parameter h, Hartmann number M, Prandtl number Pr, radiation parameter $\mathrm{N}$ and pressure gradient A as is very clear by the comparison of curves II, V, VII, VIII and IX with the dashed curve I (---). It is depicted in Fig. 4 that there is always a phase lead and it goes on increasing with increasing frequency of oscillations $\omega$.

The temperature profiles are shown in Figure 5. The figure clearly depicts that the temperature decreases with the increase of each of the parameters i.e. Prandtl number $\operatorname{Pr}$, radiation parameter $\mathrm{N}$ and the frequency of oscillations $\omega$. The amplitude $|H|$ and phase angle $\psi$ of the rate of heat transfer are shown in Figures 6 and 7 respectively. It is clear from Figure 6 that the amplitude decreases with the increase of Prandtl number and the radiation parameter. There is a sharp decrease in amplitude for the case of water $(\operatorname{Pr}=7)$ than the case of air $(\operatorname{Pr}=0.7)$. However, the amplitude remains the same for large values of radiation for increasing frequency of oscillations $\omega$. Figure 7 shows that with increasing oscillations $\omega$ the phase angle $\psi$ of the rate of heat transfer oscillates between the phase lag and the phase lead but for increased radiation parameter there is always a phase lead and the phase angle remains linear.

\section{CONCLUSIONS}

From the discussion above following conclusions are made:-

$>$ The velocity increases with the increase of slip-flow parameter $\mathrm{h}$, Grashof number $\mathrm{Gr}$, permeability of the porous medium $\mathrm{K}$ and the favourable pressure gradient $\mathrm{A}$.

$>$ But the velocity decreases with increasing viscoelastic parameter $\gamma$, Hartmann number $\mathrm{M}$, Prandtl number $\mathrm{Pr}$, radiation parameter $\mathrm{N}$ and the frequency of oscillations $\omega$.

$>$ The amplitude of the skin-friction also increases due to the increase of all those parameters because of which velocity increases and decreases with the increase of other parameters because of which velocity decreases.

$>$ The phase angle of the skin-friction increases with increase of viscoelastic parameter $\gamma$, Grashof number $\mathrm{Gr}$, and porous medium permeability $\mathrm{K}$ but decreases with the increase of slip parameter $h$, Hartmann number $M$, Prandtl number Pr, radiation parameter $\mathrm{N}$ and pressure gradient $\mathrm{A}$.

$>$ There is always a phase lead of the skin-friction and it goes on increasing with increasing frequency of oscillations $\omega$.

$>$ The temperature decreases with the increase of each of the parameters.

$>$ The amplitude of rate of heat transfer is less in water $(\operatorname{Pr}=7)$ than in air $(\operatorname{Pr}=0.7)$.

$>$ The phase of heat transfer oscillates between the phase lag and the phase lead.

\section{References:}

[1] SINGH, A.K. and SINGH, N.P., MHD flow of a dusty viscoelastic liquid through a porous medium between two inclined parallel plates, Proc. of National Academy of Sciences India 66A (1966) 143-150. 
[2] Hayat, T., Fetecau, C. and Sajid, M., Analytic solution for MHD transient rotating flow of a second grade fluid in a porous space, Nonlinear Anal.: Real Word App. 9 (2008) 1619-1627.

[3] TIwari, A.K. and Ravi, S.K., Analytical Studies on Transient Rotating Flow of a Second Grade Fluid in a Porous Medium, Adv. Theor. Appl. Mech. 2 (1) (2009) 33 - 41

[4] ChOudhary, Rita and DAS, UTPAL JYOTI, Heat transfer to MHD oscillatory viscoelastic flow in a channel filled with porous medium, Physics Research International (2012) doi: 101155/2012/879537.

[5] GHOSH, S.K. and SHIT, G.C. Mixed Convection MHD Flow of Viscoelastic Fluid in a Porous Medium past a Hot Vertical Plate, World Journal of Mechanics 2 (2012) 262271.

[6] Choudhury, R., Purkayastha, S. and Dey, D., Visco-Elastic Free Convective Flow past a Vertical Porous Plate through a Porous Medium with Suction and Heat Source, International Journal of Statistika and Mathematika 5 (2) (2013) 31-38.

[7] RAPTIS, A.A. and TAKHAR, H.S., Heat transfer from flow of an elastico-viscous fluid, Int. comm. Heat and mass transfer 16 (1989) 193-917.

[8] Hayat, T., Hutter, K. and Siddiqui, A.M., MHD Flows of an Oldroyd-B Fluid, Mathematical and Computer Modelling 36 (2002) 987-995.

[9] MEHTA, K.N. and RAO, K.N., Buoyancy induced flow of a non-Newtonian fluids over a non-isothermal horizontal plate embedded in a porous medium, Int. J. Eng. Sci. 32 (1994) 521-525.

[10] MehtA, K.N. and RaO, K.N., Buoyancy induced flow of non-Newtonian fluids in a porous medium past a vertical plate with non-uniform surface heat flux, Int. J. Eng. Sci. 32 (1994) 297-302.

[11] HAYAT, T. and HutTER, K., Rotating flow of a second-order fluid on a porous plate, Int. J. Non-Linear Mech. 39 (2004) 767-777.

[12] Hayat, T., Khan, M., Ayub, M. and Siddiqui, A.M., The unsteady Couette flow of second grade fluid in a layer of porous medium, Arch. Mech. 57 (2005) 405-416.

[13] RAJGOPAL, K.R. and GUPTA, A.S., An exact solution for the flow of a non-Newtonian fluid past an infinite porous plate. Meccanica 19 (1984) 158-160.

[14] LABROPUlu, F., Exact solution of non-Newtonian fluid flows with prescribed vorticity. Acta Mech., 141: (2000): 11-20.

[15] FeteCAU, C. and ZIEREP, J., On a class of exact solutions of the equations of motion of a second grade fluid. Acta. Mech., 150: (2001): 135-138.

[16] ARIEL, P.D., An exact solution of flow problems of a second grade fluid through two porous walls. Int. J. of Engineering Science 40: (2002): 913-941.

[17] Khan, I., ImRAn, M. and FAKHAR, K., New Exact Solutions for an Oldroyd-B Fluid in a Porous Medium, Hindawi Publishing Corporation International, Journal of Mathematics and Mathematical Sciences, Article ID 408132, (2011) 12 p.p. doi: $10.1155 / 2011 / 408132$.

[18] SingH, K.D., Viscoelastic mixed convection MHD oscillatory flow through a porous medium filled in a vertical channel, Int. J. Physical and Mathematical Sciences 3 (2012) 194-205.

[19] TICHY, J.A., Non-Newtonian lubrication with the convected Maxwell model, ASME J. Tribol. 118 (1996) 344-348.

[20] Morques, JR.W., Kermer, G.M. and Shapiro, F.M., Coutte flow with slip and jump boundary conditions, Continuum Mech. Therodynam. 12 (2000) 379-386.

[21] Rhodes, C.A. and RouleaU, W.T., Hydromagnetic lubrication of partial metal bearings, J. Basic Eng. - T. ASME 88 (1966) 53-60.

[22] Sharma, P.K. and Chaudhary, R.C., Effect of Variable Suction on Transient Free Convection Viscous Incompressible Flow Past a Vertical Plate with Periodic 
Temperature Variations in Slip-flow Regime, Emirates Journal for Engineering Research 8 (2003) 33-38.

[23] Sharma, P.K., Fluctuating Thermal and Mass Diffusion on Unsteady Free Convection Flow Past a Vertical Plate in Slip-flow Regime, Lat. Am. Appl. Res. 35 (2005) 1-12.

[24] JAIN, N.C. AND GUPTA, POONAM, Unsteady Magnetopolar Free Convection Flow in Slip Flow Regime with Variable Permeability and Constant Heat Flux, J. Energy, Heat and Mass Transfer 29 (2007) 227-240.

[25] Khaled, A.R.A. and VAfaI, K., The Effect of the Slip Condition on Stokes and Couette Flows due to an Oscillatory Wall: Exact Solution, Int. J. Nonlinear Mech. 39 (2004) 795-809.

[26] Menmood, A. and Ali, A., The effect of slip condition on unsteady MHD oscillatory flow of a viscous fluid in a planer channel, Rom. Journ. Phys. 52 (2007) 85-91.

[27] SINGH, K.D., Hydromagnetic forced convective oscillatory slip flow through porous medium in a vertical channel with thermal radiation, Proc. Indian Natn. Sci. Acad. 77 (2011) 19-30.

[28] SINGH, K.D., Solution of MHD oscillatory flow through porous medium in a vertical porous channel in slip-flow regime, J. Energy, Heat and Mass Transfer 34: (2012): 217232.

[29] Hayat, T., JAVED, T. and ABbAS, Z., Slip flow and heat transfer of a second grade fluid past a stretching sheet through a porous space, Int. J. Heat Mass transfer 51 (2008) 4528-4534.

[30] Siddiqui, A. M., Haroon, T., Zahid, M. and ShaHZad, A., Effect of Slip Condition on Unsteady Flows of an Oldroyd-B Fluid between Parallel Plates, World Applied Sciences Journal 13 (2011) 2282-2287.

[31] Ahmed, N. and TAluKDAR, S., Transient magnetohydrodynamic (MHD) flow of a visco-elastic fluid past an infinite vertical porous plate embedded in a porous medium with Hall current and slip condition in a rotating system, International Journal of Physical Sciences 7 (45) (2012) 5942-5953.

[32] OJHA, N.C. and PANDA, J.P., MHD Flow of an Elastico-viscous fluid in porous medium in a slip flow regime. Int. J. of Sci., Engineering and Technology Research (IJSETR) $\mathbf{3}$ (3) (2014) 486-490.

[33] SingH, K.D., Oscillatory MHD convective flow of a viscoelastic fluid through a porous medium in a rotating vertical channel in slip-flow regime with thermal radiation and Hall current, J. of Energy, Heat and Mass Transfer 35 (2013) 109-132.

[34] SINGH, K.D., MHD mixed convection visco-elastic slip flow through a porous medium in a vertical porous channel with thermal radiation. Kragujevac J. Sci. 35 (2013) 27-40.

[35] Coleman, B.D. and Noll, W., An approximation theorem for functional, with applications in continuum mechanics, Archive for Rational Mechanics and Analysis 6 (1960) 355-370.

[36] Markovitz, H. and Coleman, B.D., Incompressible second order fluids. Advances in Applied Mechanics 8 (1964) 69-101. 


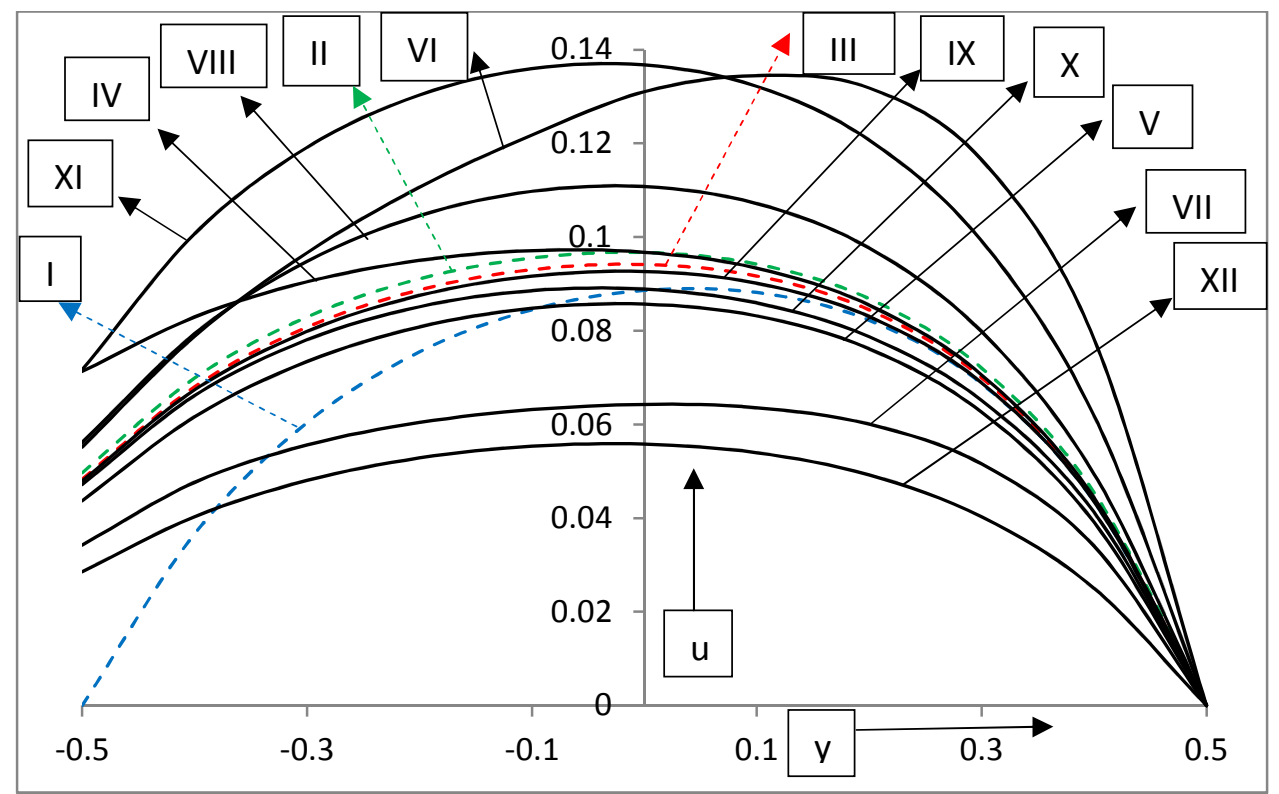

Figure 2. Velocity profiles for $\mathrm{z}=0.5$ and $\mathrm{t}=\pi / 2$.

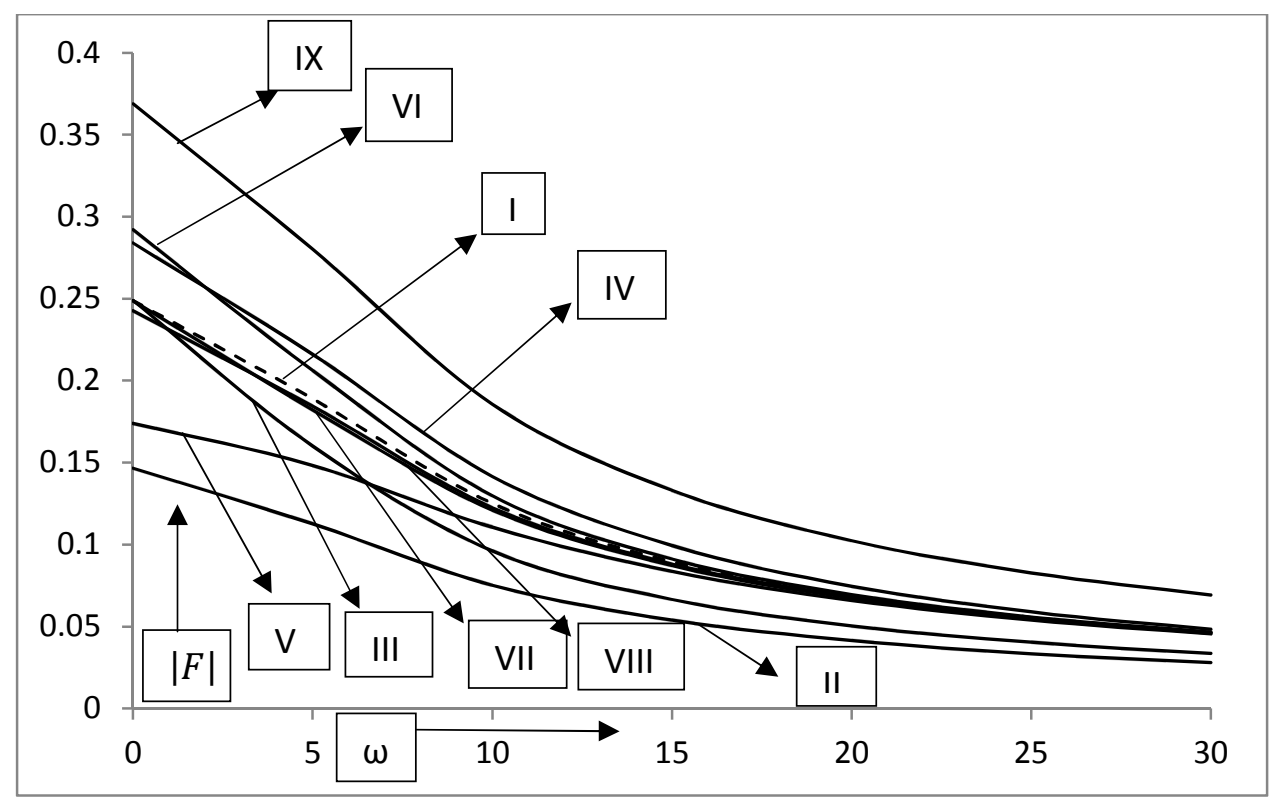

Figure 3. Amplitude of skin friction.

Table 2. Sets of parameter values plotted in Figs. 3 \& 4.

$\begin{array}{lllllllllll}\mathrm{h} & \text { V } & \mathrm{G} & \mathrm{M} & \mathrm{K} & \operatorname{Pr} & \mathrm{N} & \mathrm{A} & \text { Curves } \\ 0.2 & 0.2 & 1 & 2 & 0.2 & 0.7 & 1 & 2 & \mathrm{I}(---) \\ 0.5 & 0.2 & 1 & 2 & 0.2 & 0.7 & 1 & 2 & \mathrm{II} \\ 0.2 & 0.3 & 1 & 2 & 0.2 & 0.7 & 1 & 2 & \mathrm{III} \\ 0.2 & 0.2 & 5 & 2 & 0.2 & 0.7 & 1 & 2 & \mathrm{IV} \\ 0.2 & 0.2 & 1 & 4 & 0.2 & 0.7 & 1 & 2 & \mathrm{~V} \\ 0.2 & 0.2 & 1 & 2 & 1.0 & 0.7 & 1 & 2 & \mathrm{VI} \\ 0.2 & 0.2 & 1 & 2 & 0.2 & 7.0 & 1 & 2 & \mathrm{VII} \\ 0.2 & 0.2 & 1 & 2 & 0.2 & 0.7 & 5 & 2 & \mathrm{VIII} \\ 0.2 & 0.2 & 1 & 2 & 0.2 & 0.7 & 1 & 3 & \mathrm{IX}\end{array}$

Table 1. Sets of parameter values plotted in Fig. 2.

\begin{tabular}{|cccccccccc|}
\hline $\mathrm{h}$ & $\mathrm{V}$ & $\mathrm{Gr}$ & $\mathrm{M}$ & $\mathrm{K}$ & $\operatorname{Pr}$ & $\mathrm{N}$ & $\mathrm{A}$ & $\boldsymbol{\omega}$ & Curves \\
0 & 0.2 & 1 & 2 & 0.2 & 0.7 & 1 & 2 & 1 & $\mathrm{II}(---)$ \\
0.2 & 0 & 1 & 2 & 0.2 & 0.7 & 1 & 2 & 1 & $\mathrm{II}(---)$ \\
0.2 & 0.2 & 1 & 2 & 0.2 & 0.7 & 1 & 2 & 1 & $\mathrm{III}(---)$ \\
0.5 & 0.2 & 1 & 2 & 0.2 & 0.7 & 1 & 2 & $1 \mathrm{IV}$ \\
0.2 & 0.5 & 1 & 2 & 0.2 & 0.7 & 1 & 2 & $1 \mathrm{~V}$ \\
0.2 & 0.2 & 5 & 2 & 0.2 & 0.7 & 1 & 2 & $1 \mathrm{VI}$ \\
0.2 & 0.2 & 1 & 4 & 0.2 & 0.7 & 1 & 2 & $1 \mathrm{VII}$ \\
0.2 & 0.2 & 1 & 2 & 1.0 & 0.7 & 1 & 2 & $1 \mathrm{VIII}$ \\
0.2 & 0.2 & 1 & 2 & 0.2 & 7.0 & 1 & 2 & 1 & $\mathrm{IX}$ \\
0.2 & 0.2 & 1 & 2 & 0.2 & 0.7 & 5 & 2 & $1 \mathrm{X}$ \\
0.2 & 0.2 & 1 & 2 & 0.2 & 0.7 & 1 & 3 & $1 \mathrm{XI}$ \\
0.2 & 0.2 & 1 & 2 & 0.2 & 0.7 & 1 & 2 & $5 \mathrm{XII}$ \\
\hline
\end{tabular}




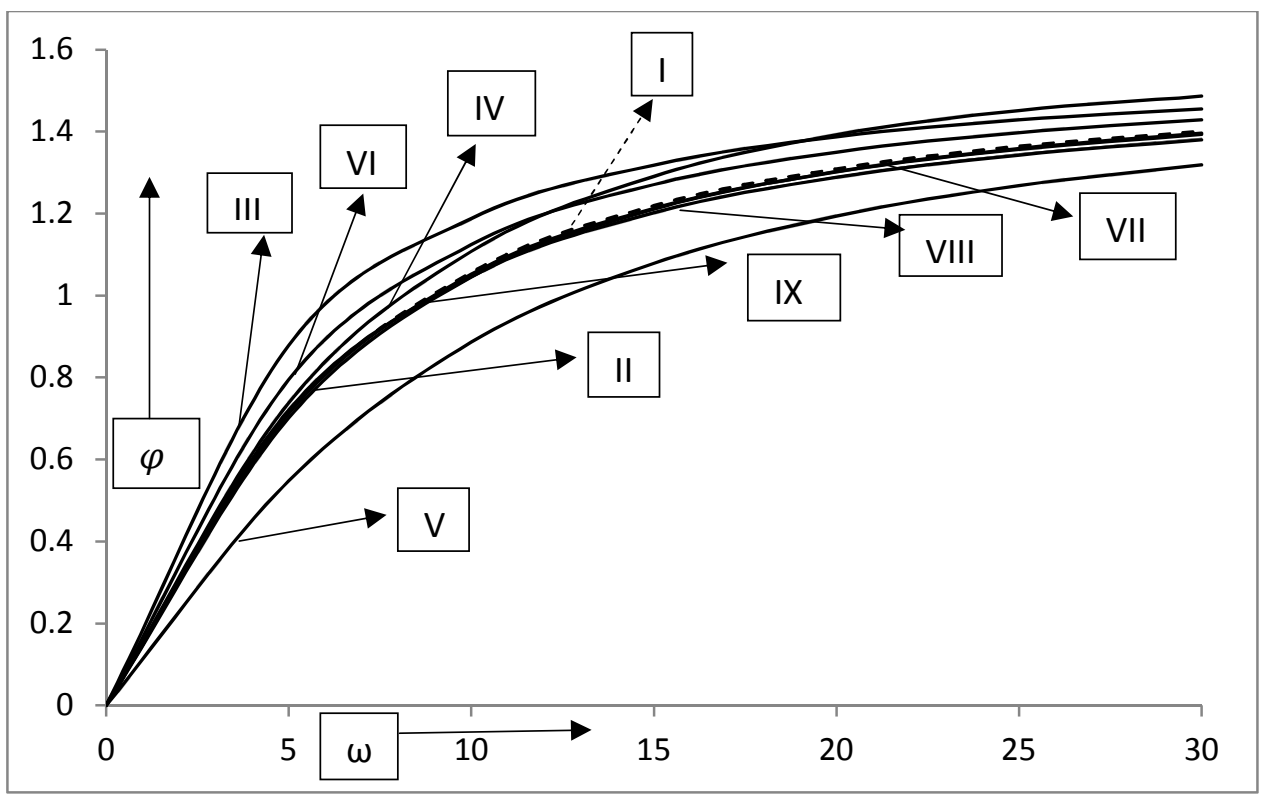

Figure 4. Phase angle of the skin-friction.

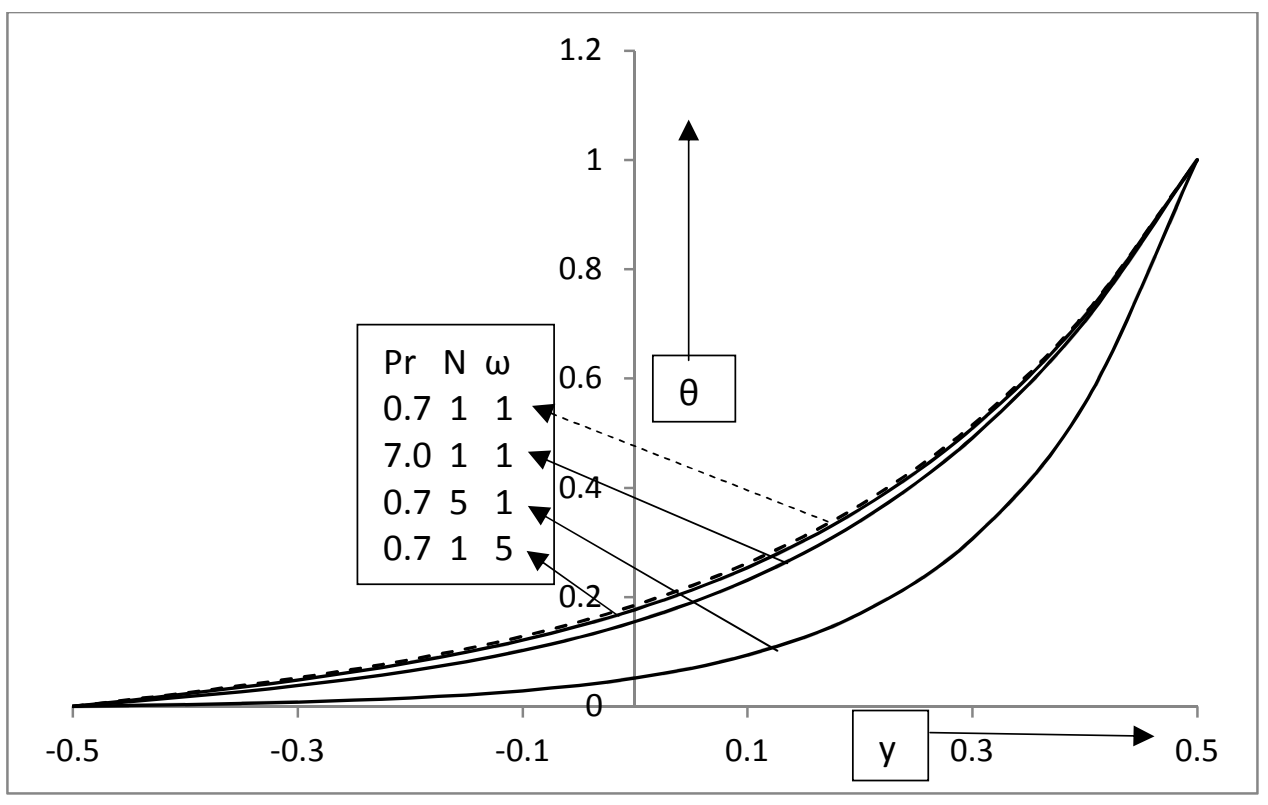

Figure 5. Temperature profiles for $\mathrm{z}=0.5$ and $\mathrm{t}=\pi / 2$. 


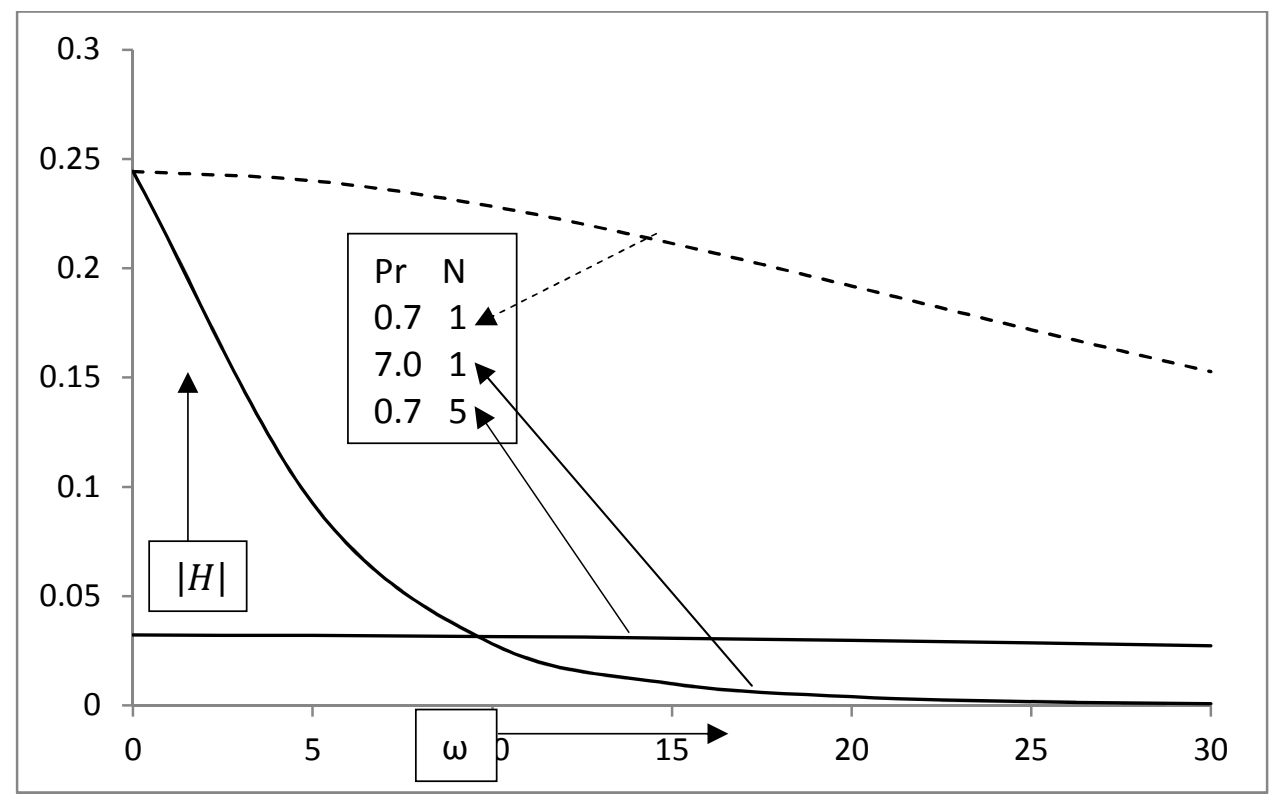

Figure 6. Amplitude of Nusselt number.

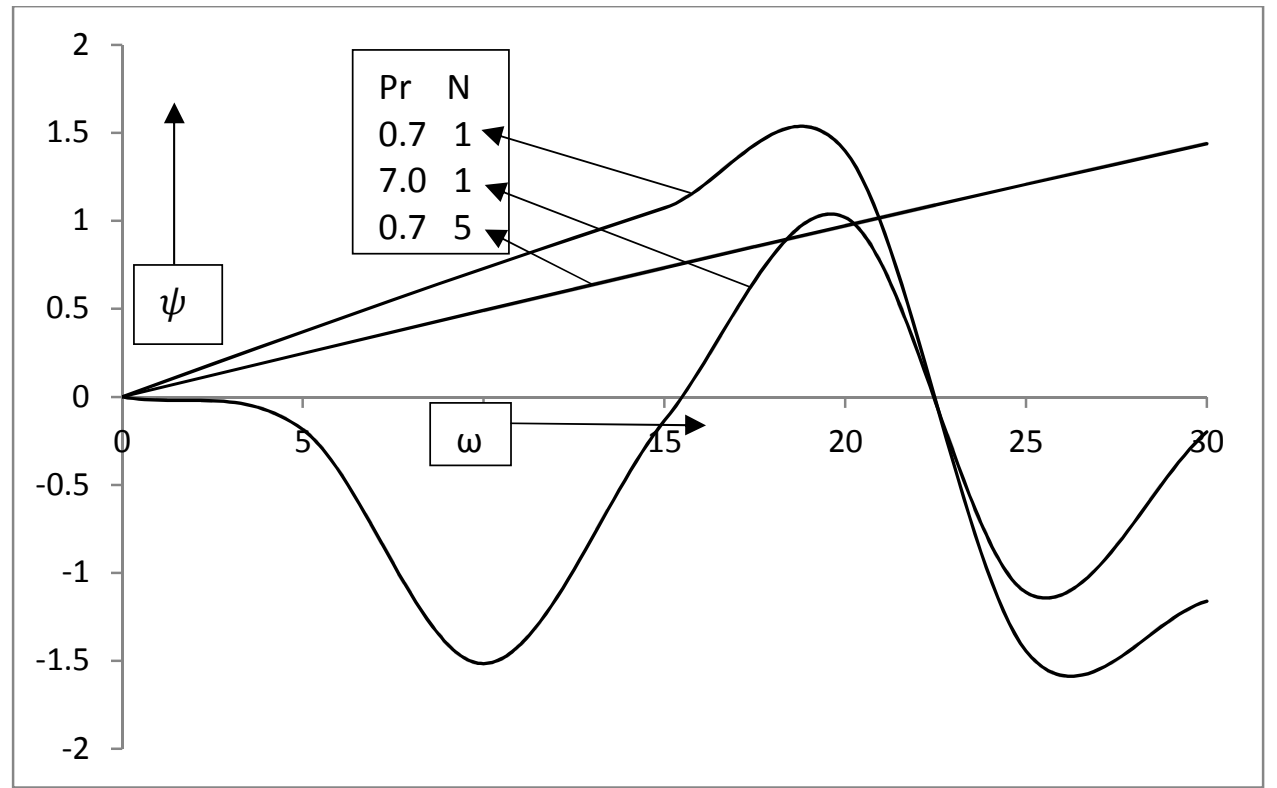

Figure 7. Phase angle of Nusselt number. 